\title{
InP Membrane Based Broadband Regenerator for silicon-based optical interconnect applications
}

\author{
O. $\operatorname{Raz}^{1}$, M. Tassaert ${ }^{2}$, G. Roelkens ${ }^{2}$, H.J.S. Dorren ${ }^{1}$ \\ 1:Eindhoven University of Technology, Den Dolech 2, 5600MB, Eindhoven, The Netherlands \\ 2:INTEC, Ghent University—IMEC, St-Pietersnieuwstraat 41, 9000 Ghent, Belgium
}

Abstract: We demonstrate the use of a Membrane-InP-Switch(MIPS) on-silicon as a signal regenerator. A receiver sensitivity enhancement $>2.5 \mathrm{~dB}$ across the entire C-band and a tripling of Extinction Ratio(ER) for low ER signals at $1 \mathrm{~Gb} / \mathrm{sec}$ is demonstrated.

OCIS codes: (130.3120) Integrated optics devices; (200.6015) Signal regeneration

\section{Introduction}

Silicon photonics has seen increasing interest from industry [1,2] and academia [3-5] as a possible ultimate merging of CMOS and photonics on a unified platform. The basic passive building blocks needed to make integrated photonic circuits have been recognized already 20 years ago [6,7] and were demonstrated in recent years. Complementary functions such as light sources and detectors have also been achieved with the most successful demonstrations based on the heterogeneous/hybrid integration of III-V materials on top of silicon [4,5]. Yet another critical component being pursued is the electrical to optical convertor or modulator [8]. Demonstrated silicon modulators are limited by the properties of carriers in doped silicon and have therefore branched along two clear paths. For the realization of high speed and low voltage devices, solutions based on resonant cavities, and therefore narrow band in nature, have been used [9]. Where broad band operation was desired, Mach Zehnder Interferometer (MZI) structures have been built with typically a $V \pi L$ product of around $1-2 \mathrm{~V} \cdot \mathrm{cm}$ implying that modulators need either be very long or driven with high voltages in order to achieve a desired high extinction ratio (ER). Limiting the length and/or voltage of the MZI modulators results in poor ER, which in turn lowers the receiver sensitivity. It is therefore desirable to find a low power method for increasing the ER (regeneration) in the silicon circuit. Recently we have reported on a new device based on an ultra thin Membrane InP Switch on Silicon called the MIPS [10] which can precisely fulfill this function.

In this paper we demonstrate how the MIPS can be used as a passive signal regenerator for boosting the ER of low ER signals through the principal of saturable absorption. Tripling of the ER for low ER signals at $1 \mathrm{~Gb} / \mathrm{sec}$ is shown and lowering of the required power at the receiver by more than $2.5 \mathrm{~dB}$ is shown across the C-band. The demonstrated performance enhancement achieved with the heterogeneously integrated MIPS device on an SOI platform can enable the shortening of MZI Si structures or alternatively the lowering of their required modulation driving voltages.

\section{MIPS description and typical characteristics}

The InP membrane switch is made by adhesive bonding of a thin $(<100 \mathrm{~nm})$ III-V layer consisting of 3 quantum wells (QWs) and further post-processing of the membrane to leave a $2 \mu \mathrm{m}$ wide and $150 \mu \mathrm{m}$ long InP stripe on top of an $\mathrm{SiO}_{2}$ dielectric. The optical signals are horizontally coupled using a polymer inverted taper coupler into the SOI and then transferred from the SOI waveguide to the InP switch structure by using an inverted taper with appropriate lengths in both the SOI and InP layers.

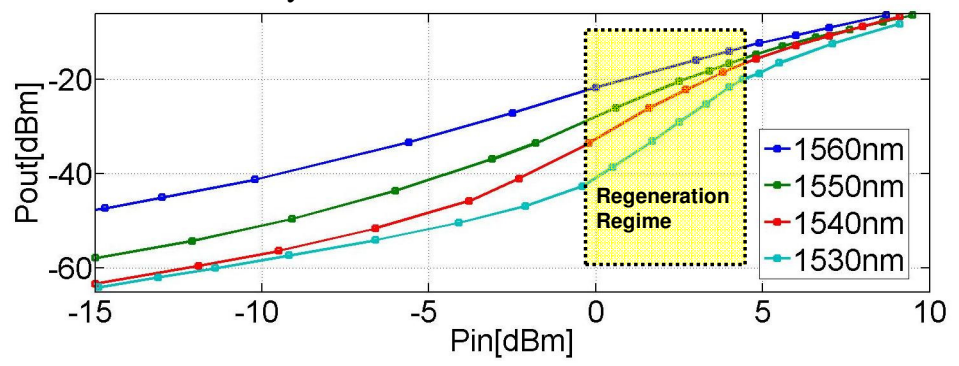

Figure 1: MIPS Pin-Pout characteristics showing the large slope in the regeneration regime

The InGaAs QWs are designed with a band gap of $1.58 \mu \mathrm{m}$. Light absorption is critically dependent on the wavelength [10] and since the membrane has such a small volume (QW layers: $7.2 \mu \mathrm{m}^{3}$, total structure: $30 \mu \mathrm{m}^{3}$ ) the $P_{\text {in }}$ to $P_{\text {out }}$ characteristics exhibit two distinct regimes (see figure 1). For low optical powers linear absorption dominates the behavior while for high input power, quick bleaching on the material results in a slope larger than one and hence a regeneration action. In addition, the further away from the band-edge the more pronounced the slope becomes in the regeneration regime. 


\section{MIPS operation as regenerator}

From the slopes in figure 1 it is visible that in the regeneration regime a $\Delta$ Pin to $\Delta$ Pout ratio of 3:1 or more can be obtained. This suggests that the extinction ratio can be greatly increased by injecting a small ER signal with the appropriate optical power (as can also be seen in figure 2C). To test the MIPS regenerator an optical input signal modulated with a $2^{31}-1$ PRBS sequence and varying extinction ratios was used. Two different scenario's were tested: operation at a $2 \mathrm{~dB}$ input ER (figure 2A) and operation at $5 \mathrm{~dB}$ ER (figure 2B). For both cases the measured receiver sensitivity can be improved by at least $2.5 \mathrm{~dB}$ with the best results achieved for $2 \mathrm{~dB}$ input ER at $1530 \mathrm{~nm}$ where the obtained receiver sensitivity improvement was $4.5 \mathrm{~dB}$. Additional characterization (not included) showed that even steeper Pin Vs Pout curves can be obtained for shorter wavelengths, Therefore a QW epitaxy engineered with a band edge at $1.6 \mu \mathrm{m}$ for example should yield $>4 \mathrm{~dB}$ enhancement across the $\mathrm{C}$-band.
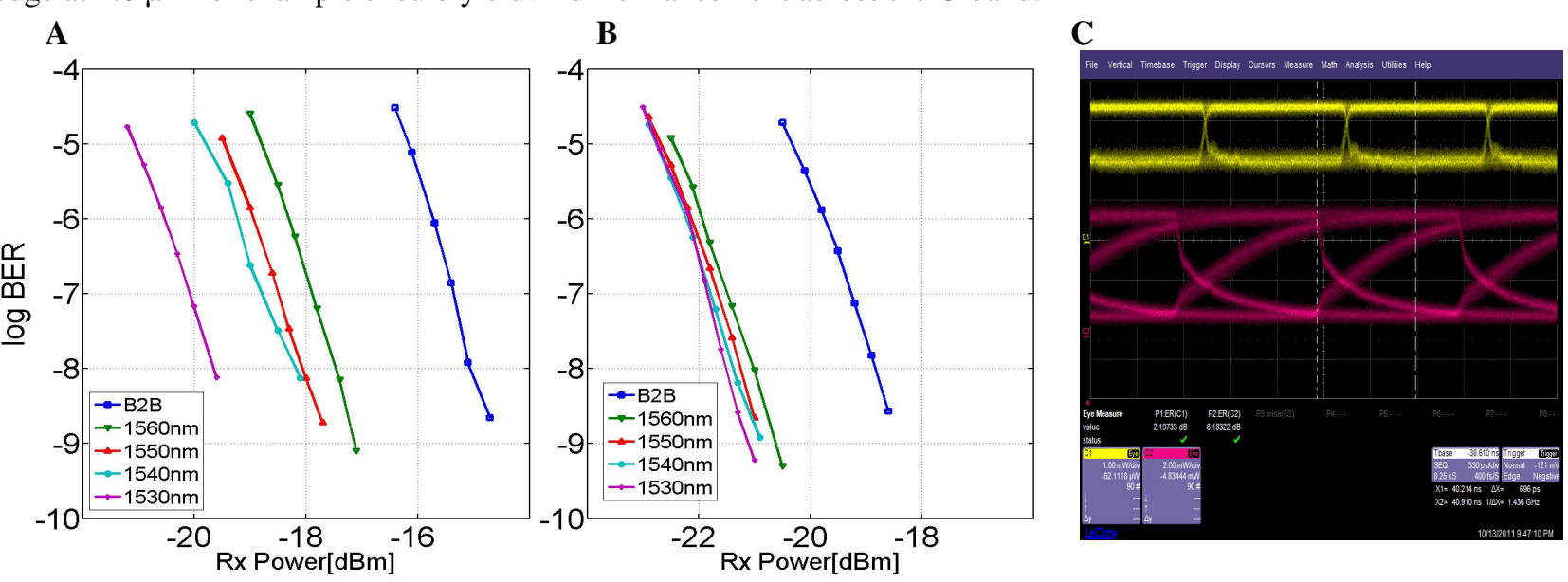

Figure 2: (A) Measured BER Vs Rx power for an input ER of 2dB; (B) Measured BER Vs Rx power for an input ER of 5dB; (C)

$3 \mathrm{x}$ ER improvement at $1530 \mathrm{~nm}$

\section{Discussion}

It is clear that the operation speed of the device is limited by the carrier lifetime. The effective carrier lifetime can be decreased by sweeping the carriers out of the active region through the application of an electrical field. This can be done either in a p-i-n configuration or in a metal-semiconductor-metal configuration. Alternatively, the carrier lifetime can be reduced by ion implantation in the active regions. Reducing the carrier lifetime of course has an adverse effect on the required signal power for substantial bleaching of the multi-quantum well absorption, which is an intrinsic trade-off for this device.

\section{Conclusions}

We demonstrated the tripling of the ER for low ER signals using a passive MIPS device. ER improvements from $2 \mathrm{~dB}$ to $6 \mathrm{~dB}$ and from $5 \mathrm{~dB}$ to $13 \mathrm{~dB}$ at $1 \mathrm{~Gb} / \mathrm{sec}$ were measured and the resulting receiver sensitivity has been improved by at least $2.5 \mathrm{~dB}$ across the C-band. All values mentioned in this paper are for optical powers in the fiber leading to and from the MIPS device, which indicates that the regeneration action occurs in the chip for submilliwatt power levels. Many options exist to reduce the carrier lifetime which would pave the way to higher speed operation.

\section{References}

[1] A. Barkai et al, "Integrated silicon photonics for optical networks [Invited]", J. of Optical Networking, Vol. 6, No. 1, pp. 25-47, 2007

[2] Y. Vlasov, "Silicon Photonics for next generation computing systems [Tutorial]", Proc. of ECOC 2008, SC2, 2008

[3] Q. Xu et al, "12.5 Gbit/s carrier-injection-based silicon microring silicon modulators", Opt. Exp. Vol. 15, No.2, pp.430-436, 2007

[4] A. W. Fang et al, "Electrically pumped hybrid AlGaInAs-silicon evanescent laser", Vol. 14, no. 20, pp.9203-9210, 2006

[5] D. Van Thourhout et al, IEEE JSTQE, vol. 16, No. 5, pp.1363-1375, 2010

[6] I. Belz et al, Mater. Sci. Eng. ,Vol. 34, pp. 429-433, 1989

[7] J. Schmidtchen et al, "low loss singlemode optical waveguides with large corss-section in silicon on insulator", Elec. Lett., Vol. 27, No.16, pp.1486-1487, 1992

[8] G. T. Reed et al, Nature Photonics, Vol. 4, pp.518-526, August 2010

[9] G. Li et al," 25Gb/s 1V-driving CMOS ring modulator with integrated thermal tuning”, Opt. Exp. Vol.19, no.21, pp.20435-20443, 2011

[10]O. Raz et al, "Bias-Free, Low Power and Optically Driven InP on SOI Switch for Remotely Configurable Photonic Packet Switches", proc. of ECOC 2011, Th13A4, 2011(http://www.miracd.com/ECOC2011/files/Th.13.A.4.pdf) 